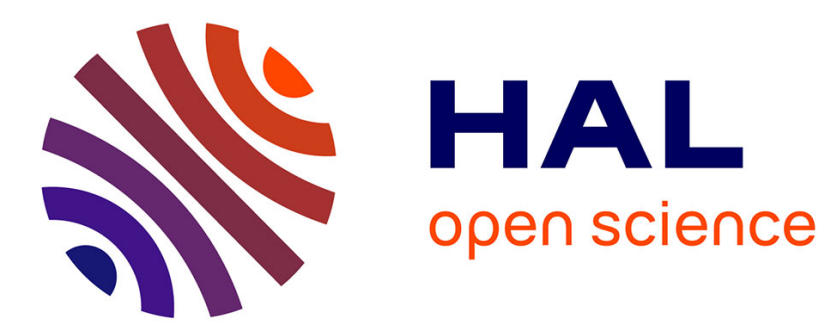

\title{
Orientation d'un smectique à différentes interfaces et structures induites par celles-ci
}

Emile Perez, J.E. Proust

\section{To cite this version:}

Emile Perez, J.E. Proust. Orientation d'un smectique à différentes interfaces et structures induites par celles-ci. Journal de Physique Lettres, 1977, 38 (4), pp.117-120. 10.1051/jphyslet:01977003804011700 . jpa-00231339

\section{HAL Id: jpa-00231339 https://hal.science/jpa-00231339}

Submitted on 1 Jan 1977

HAL is a multi-disciplinary open access archive for the deposit and dissemination of scientific research documents, whether they are published or not. The documents may come from teaching and research institutions in France or abroad, or from public or private research centers.
L'archive ouverte pluridisciplinaire HAL, est destinée au dépôt et à la diffusion de documents scientifiques de niveau recherche, publiés ou non, émanant des établissements d'enseignement et de recherche français ou étrangers, des laboratoires publics ou privés. 


\title{
ORIENTATION D'UN SMECTIQUE A DIFFÉRENTES . INTERFACES ET STRUCTURES INDUITES PAR CELLES-CI
}

\author{
E. PEREZ et J. E. PROUST \\ Physico-chimie des surfaces et des membranes, UER Biomédicale, \\ 45, rue des Saints-Pères, 75270 Paris cedex 06, France
}

(Reçu le 6 décembre 1976. révisé le 14 janvier 1977, accepté le 19 janvier 1977)

\begin{abstract}
Résumé. - On étudie l'orientation des molécules d'octylcyanobiphényl (8 CB), en phase nématique et smectique à différentes interfaces : cristal liquide-air, cristal liquide-eau et cristal liquideverre traité par une couche monomoléculaire de polyélectrolyte. Dans ce dernier cas, on peut obtenir une très bonne orientation planaire uniaxe en phase smectique.
\end{abstract}

\footnotetext{
Abstract. - We have observed the orientation of octylcyanobiphenyl (8 CB) in the nematic and smectic phases at several interfaces : liquid crystal/air, liquid crystal/water, liquid crystal/glass covered with a monolayer of polyelectrolyte. In the last case, we can produce a good planar uniaxial anchorage of the smectic phase.
}

1. Introduction. - Nous nous proposons, dans ce court article de présenter quelques résultats expérimentaux obtenus dans l'étude d'un cristal liquide présentant une phase smectique $\mathrm{A}$ à basse température : l'octylcyanobiphényl $(8 \mathrm{CB})$.<smiles>CCCCCCCCCCCCCCC1CCC2(CCC(C#N)CC2)CC1</smiles>

solide $\rightleftarrows$ smectique $\mathrm{A} \rightleftarrows$ nématique $\rightleftarrows$ isotrope $21^{\circ} \quad 32^{\circ} \quad 39^{\circ}$

Cette étude concerne :

1. L'orientation aux surfaces libres (film mince symétrique).

2. L'orientation à l'interface CL-eau (film mince asymétrique).

3. L'ancrage sur une surface solide traitée.

Elle fait suite à une série d'études effectuées sur les cristaux liquides nématiques et plus spécialement sur le pentylcyanobiphényl (5 $\mathrm{CB})$, analogue du 8 CB $[1,2]$.

2. Surfaces libres. - L'orientation des molécules de 8 CB aux surfaces libres est déduite de l'observation au microscope polarisant d'un film mince smectique. Les films minces sont formés dans des cellules cylindriques de diamètre 1 à $5 \mathrm{~mm}$ (Fig. 1). Un piston de mercure permet de pomper le liquide qui forme dans la cellule une goutte biconcave et de former au centre un film dont le diamètre et l'épaisseur peuvent être contrôlés. L'étude d'un tel
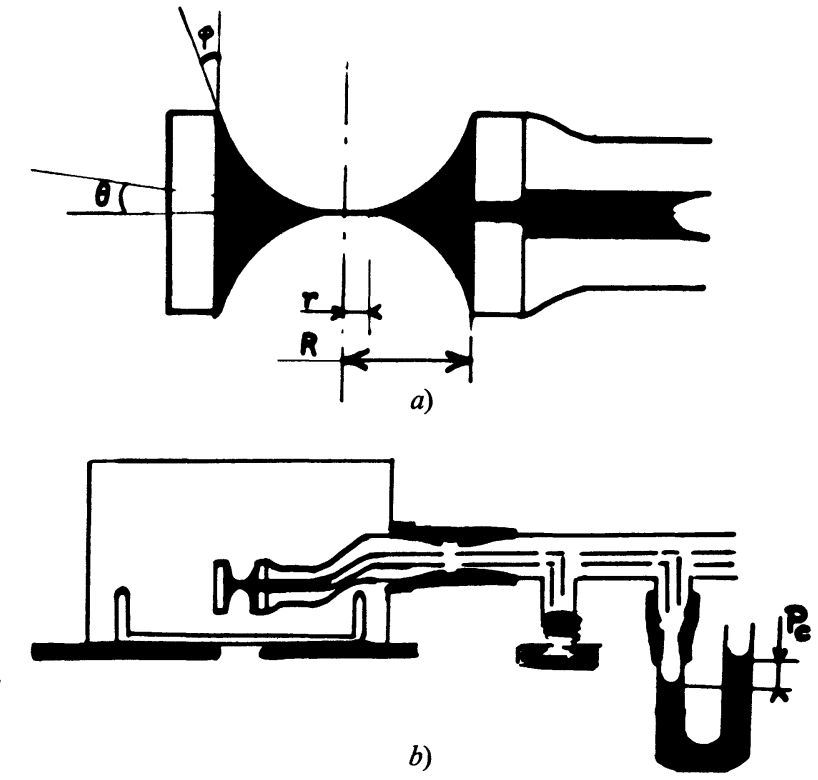

Fig. 1. - a) Cellule pour l'étude des films symétriques ou asymétriques de cristal liquide. $r$ : rayon du film, $R$ : rayon de la cellule cylindrique, $\theta$ : angle de raccordement du ménisque avec la cellule, $\varphi$ : angle de raccordement du film avec le ménisque. $b$ ) Dispositif de mesure permettant l'observation du film par réflection et transmission. La cuve en verre optique peut être remplie d'un deuxième liquide.

[a) Cell for symmetrical or asymmetrical liquid crystal film study. $r$ : film radius, $R$ : cylindrical cell radius, $\theta$ : film-meniscus contact angle, $\varphi:$ meniscus-cell contact angle. $b$ ) System allowing the observation of the film in reflection or transmission. The glass container can be filled with another liquid.] 
film smectique fait l'objet d'un deuxième article $[2 b]$, ici nous indiquerons seulement que pour des épaisseurs variant depuis $50 \AA$ jusqu'à plus de $10 \mu \mathrm{m}$, la structure est parfaitement homéotrope (Fig. 2).

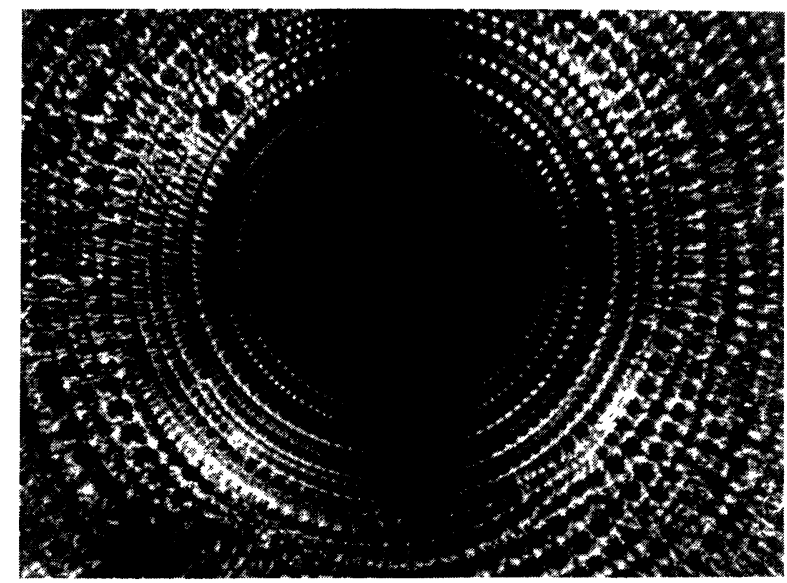

Fig. 2. - Film libre de $8 \mathrm{CB}$ observé entre polariseurs croisés. Au centre, le film est plan parallèle et homéotrope, dans le ménisque, on observe les gradins décorés en perles.

[Free $8 \mathrm{CB}$ film observed between crossed polarisers. In the centre, the film is flat and the liquid crystal orientation is homeotropic; the meniscus exhibits pearl-like stepped decoration.]

Cette orientation des molécules, perpendiculaire à la surface libre que nous trouvons pour le $8 \mathrm{CB}$, aussi bien en phase nématique qu'en phase smectique a aussi été observée sur le 5 CB [1]; D. Langevin trouve pour ce même CL la même orientation en phase nématique, par contre, en phase smectique, cet auteur trouve que les molécules font un angle avec la normale [3].

3. Interface CL-eau. - Nous avons décrit précédemment $[1,2]$ les structures observées sur un film mince de 5 CB déposé à la surface de l'eau. La structure du $8 \mathrm{CB}$ en phase nématique est tout à fait analogue; les molécules de $8 \mathrm{CB}$ sont parallèles à la surface de l'eau et perpendiculaires à la surface libre.

En phase smectique, on obtient la structure en polygones de la figure 3 , du moins pour des épaisseurs supérieures à $h_{\mathrm{c}}=1 \mu \mathrm{m}$.

Ce smectique présente une transition $\mathrm{N} \rightarrow \mathrm{Sm}$ du second ordre [3]. On observe une structure transitoire en chevrons due aux ancrages dissymétriques. On sait [5] que la structure finale est nucléee par les défauts de cette structure intermédiaire; cependant dans notre cas, la structure finale obtenue ne dépend aucunement de l'histoire de l'échantillon. La formation, ou l'amincissement du film, peut aussi bien être faite en phase isotrope, nématique ou smectique.

L'observation sans polariseurs (Fig. 3a) montre des lignes de raccordement des plans smectiques à l'interface CL-eau et des points singuliers à l'interface CL-eau. L'observation entre polariseurs croisés

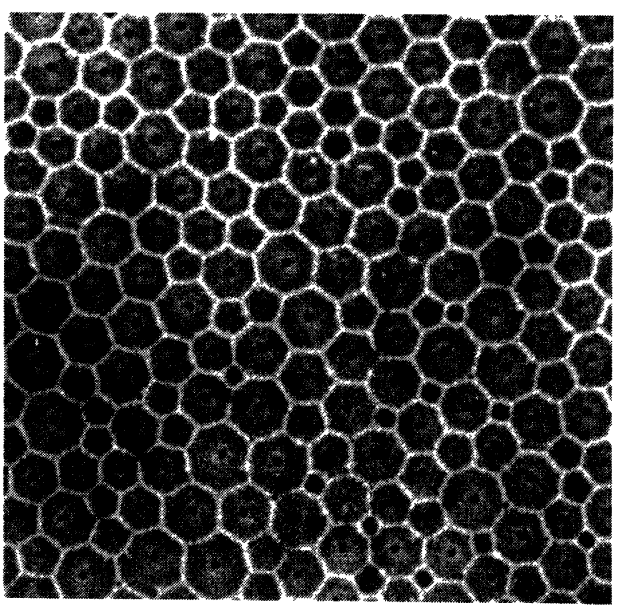

a)
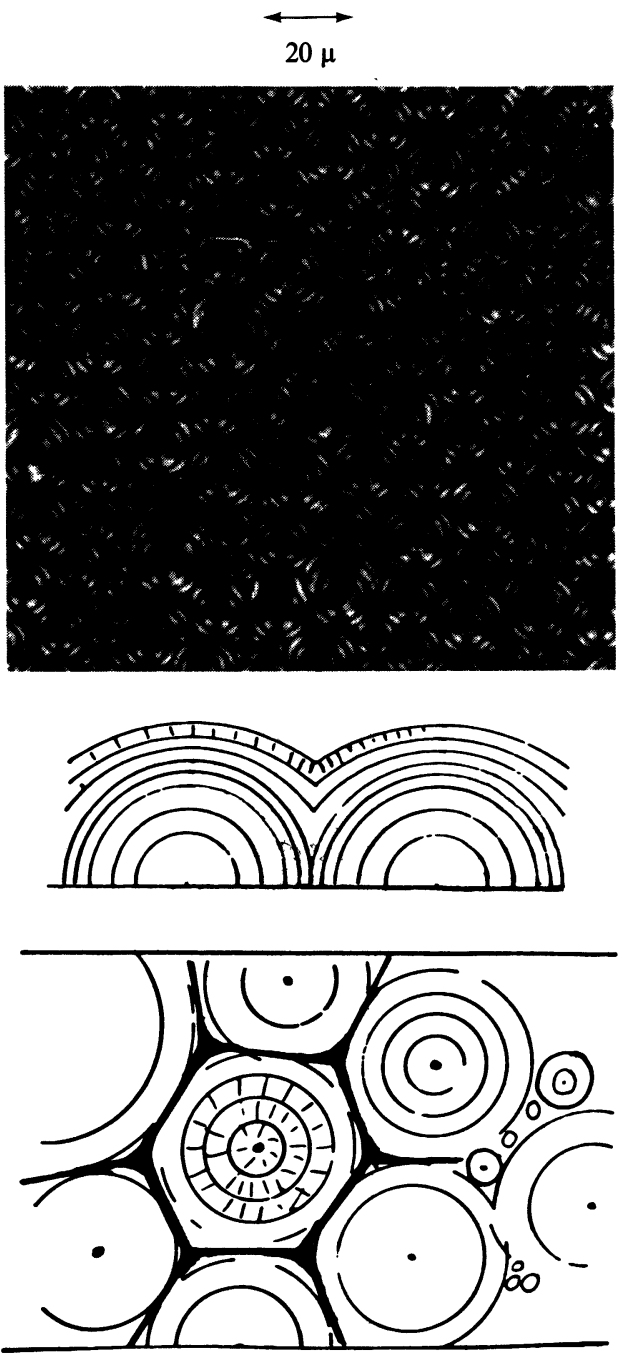

Fig. 3. - Film de $8 \mathrm{CB}$ smectique sur l'eau. $\left(P_{\mathrm{c}}=0, h=5 \mu \mathrm{m}\right.$.) a) Observation en lumière naturelle $\{$ mise au point à

b) Observation entre polariseurs croisés l'interface C.L.-eau.

c) Schéma des plans smectiques, coupe verticale.

d) Schéma des plans smectiques, coupe horizontale.

[8 CB smectic film on water $\left(P_{\mathrm{c}}=0, h=5 \mu \mathrm{m}\right)$

a) Observation in natural light $\quad$ Focussed at liquid

b) Observation between crossed polarisers $\left\{\begin{array}{l}\text { crystal/water interface } \\ \text { croction }\end{array}\right.$

c) Structure of smectic layers : vertical section.

d) Structure of smectic layers : horizontal section.] | 

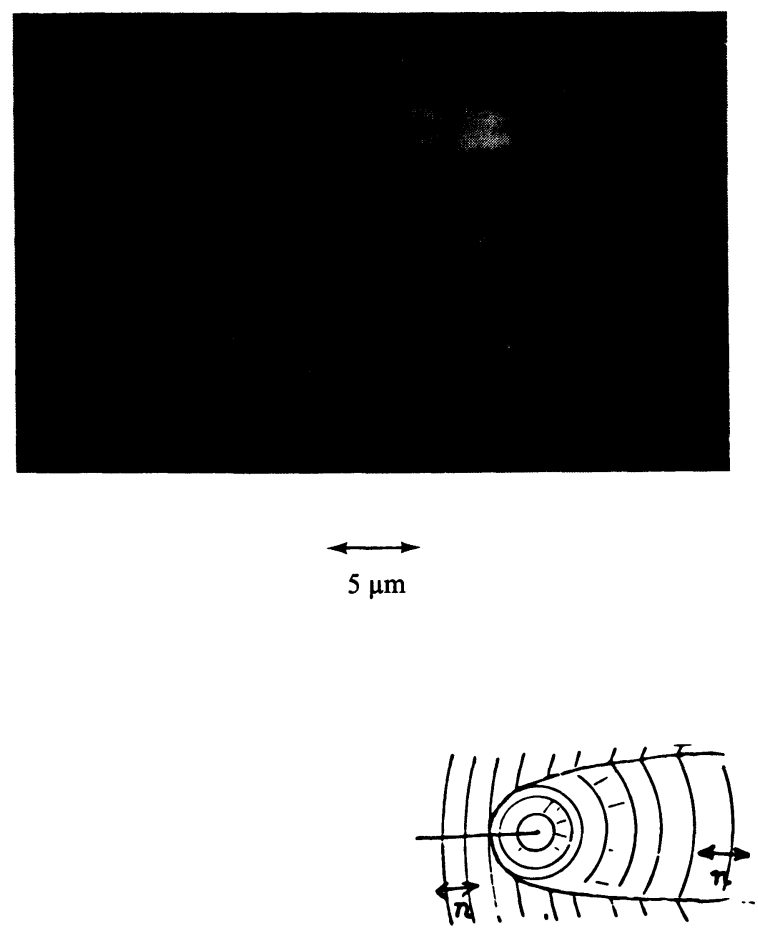

b)

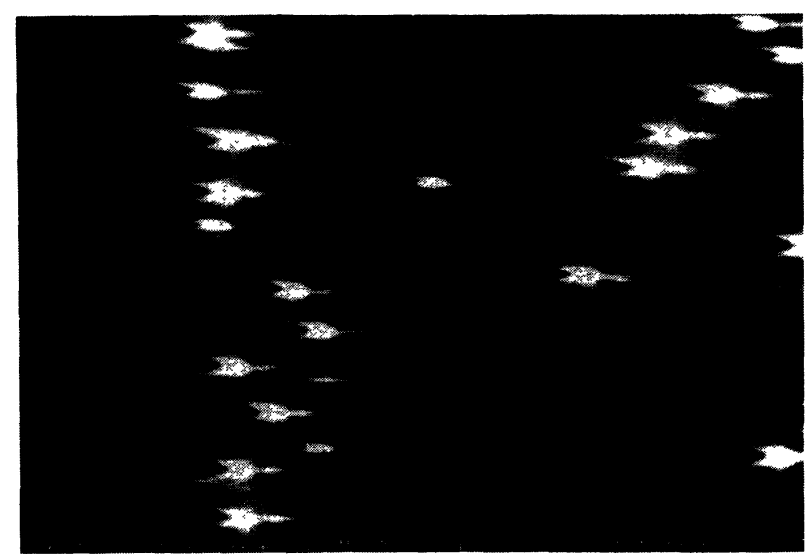

a)

$\longleftrightarrow$

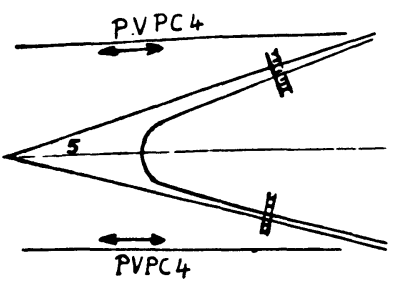

c)

FIG. 4. - Smectique planaire entre deux lames traitées par $\mathrm{PVPC}_{4}$. a) Observation entre polariseurs croisés.

b) Schéma des plans smectiques, coupe horizontale. c) Schéma des plans smectiques, coupe verticale.

[Planar smectic between two glass slides covered by a monolayer of PVPC $_{4}$. a) Observation between crossed polarisers.

b) Structure of smectic layers : horizontal section. $c$ ) Structure of smectic layers : vertical section.]

(Fig. 3b) montre dans chaque polygone une série d'anneaux de Newton semblables à ceux qu'on pourrait observer pour une lentille biconcave : la différence de marche est minimale au centre. La plupart des polygones ont un rayon égal à l'épaisseur du film. A partir de ces observations nous proposons, pour le film smectique, la structure présentée sur les schémas (Fig. 3c, 3d) correspondant à un ancrage planaire sur l'eau et homéotrope à la surface libre. Nous verrons en 4 qu'une structure très semblable peut être obtenue entre deux lames traitées de façon à obtenir des ancrages planaires et homéotropes respectivement.

Les contraintes imposées par les deux surfaces nécessitent la considération de deux problèmes : le remplissage de l'espace et la forme de la surface libre.

On a observé (Fig. 3) deux types de remplissage différents : par déformation des couches smectiques, on obtient à l'interface eau-C.L. des polygones (ce type de remplissage est ici le plus fréquent). On observe parfois un remplissage itératif.

A la surface libre il y a compétition entre une déformation de la surface qui conserverait l'ancrage homéotrope et une inclinaison des molécules qui permettrait de laisser la surface libre non déformée. On peut évaluer l'ordre de grandeur des excès d'énergie superficielle correspondant à ces deux éventualités.
La comparaison de $\gamma \Delta S$ et de $S \Delta \gamma$ permet une distorsion de la surface d'amplitude $h$ de l'ordre de

$$
h \simeq R \sqrt{\Delta \gamma / \gamma}
$$

si $R$ est le rayon des domaines.

Avec $R=5 \mu \mathrm{m}, \gamma=40$ ergs. $\mathrm{cm}^{-2}$ et

$$
\Delta \gamma>2,5 \times 10^{-2} \mathrm{erg} . \mathrm{cm}^{-2} \text {, }
$$

l'ordre de grandeur de $h$ est $1000 \AA$.

Pour de très faibles épaisseurs du film smectique déposé sur l'eau, on observe une transition structure en polygones $\leftrightarrows$ structure homéotrope. Tout se passe comme si l'ancrage homéotrope imposé à l'interface C.L.-air était plus important que l'ancrage planaire qui existe à l'interface C.L.-eau, contrairement à ce que nous avons trouvé pour le $5 \mathrm{CB}$.

En phase nématique on observe le même basculement pour une épaisseur critique $h_{\mathrm{c}}^{\prime} \simeq 0,3 \mu \mathrm{m}$. L'énergie d'ancrage correspondante (interface C.L.-eau) est $2,5 \times 10^{-2} \mathrm{erg} . \mathrm{cm}^{-2}$.

4. Ancrage sur une surface solide traitée. - Nous avons montré précédemment qu'une couche monomoléculaire de bromure d'alkylpolyvinylpyridinium $\left(\mathrm{PVPC}_{n}\right)$ prélevée sur une lame de verre permet d'obtenir, selon la longueur de la chaîne aliphatique et selon la densité superficielle, un ancrage planaire ou homéotrope pour $5 \mathrm{CB}$ [4]. 
En phase nématique, $8 \mathrm{CB}$ se comporte de la même façon : nous avons ainsi pu obtenir une très bonne orientation homéotrope avec $\mathrm{PVPC}_{16}$ et planaire avec $\mathrm{PVPC}_{4}$. Si on refroidit le $5 \mathrm{CB}$ en phase smectique, l'ancrage est conservé.

Dans le cas de la structure homéotrope, on ne peut discerner au microscope aucun défaut.

Dans le cas de la structure planaire, on sait [5] qu'il est très difficile, si on ne dispose pas d'un champ magnétique, et si on ne refroidit pas extrêmement lentement l'échantillon, d'obtenir une structure sans

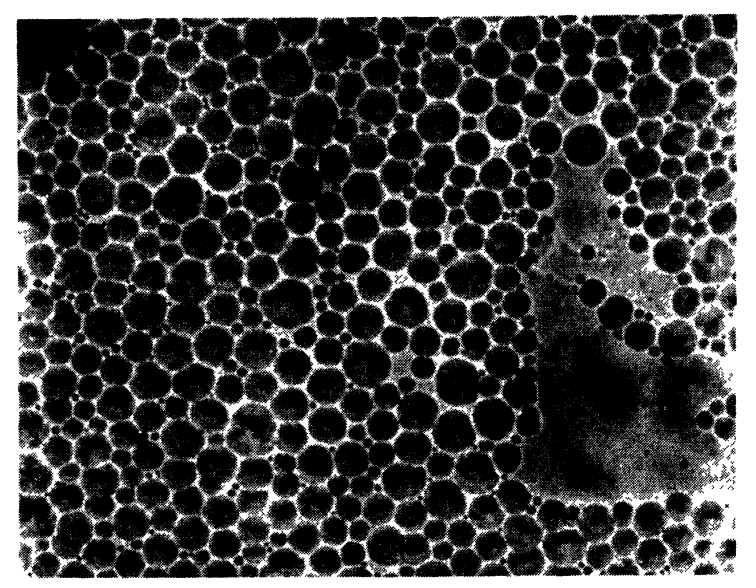

FIG. 5. - Smectique $8 \mathrm{CB}$ entre deux lames traitées par PVPC et PVPC $_{16}$.

[Smectic $8 \mathrm{CB}$ between two glass slides : one is covered with $\mathrm{PVPC}_{4}$, the other with PVPC $_{16}$.]

défauts. Ne disposant ni de l'un ni de l'autre nous obtenons une structure qui semble à l'œil très bien ordonnée et qui au microscope présente l'aspect de la figure 4. Les seuls défauts observés sont des réseaux de coniques focales très fines et très allongées selon l'axe planaire du C.L. et situé dans la masse. Ces ellipses ont un petit axe $b$ égal à la moitié de l'épaisseur de l'échantillon. L'excentricité mesurée est typiquement égale à 0,99 . L'angle que font les asymptotes de l'hyperbole conjuguée avec l'axe planaire est égal à $5^{\circ}$. On voit que dans ces conditions, les molécules de $8 \mathrm{CB}$ ancrées aux surfaces font un très petit angle avec l'axe planaire. Il n'est pas exclu que le raccordement soit tangentiel.

A partir du même échantillon en phase nématique, l'observation de parois de Néel localisées sur une surface [6] permet de déterminer l'énergie d'ancrage par rapport à une rotation de vecteur normal à l'interface

$$
w_{\mathrm{s}}=h K \pi^{2} / 4 d^{2} \simeq 0,2 \mathrm{erg} \cdot \mathrm{cm}^{-2}
$$

avec $d$ (largeur de la paroi) $1,5 \mu \mathrm{m}$.

Nous n'avons pas pu évaluer l'énergie d'ancrage par rapport à une rotation de vecteur coplanaire à l'interface : absence de parois de Bloch. Cette énergie doit être importante [7].

Pour vérifier la structure proposée (Fig. 3) pour le film smectique déposé sur l'eau, nous avons réalisé le modèle suivant : on forme une couche de $8 \mathrm{CB}$ entre deux lames traitées respectivement avec $\mathrm{PVPC}_{4}$ et $\mathrm{PVPC}_{16}$; on obtient en phase nématique une orientation moyenne inclinée à $45^{\circ}$. En phase smectique, on obtient la structure représentée figure 5 . Cette structure est très voisine de celle obtenue sur l'eau quoiqu'elle soit moins régulière. L'observation entre polariseurs croisés ne permet plus de déceler l'axe planaire.

Remerciements. - Nous sommes très reconnaissant à L. Ter Minassian Saraga pour de nombreux conseils et discussions.

Bibliographie

[1] Perez, E., Proust, J. E., Ter Minassian Saraga, L., Communication à $6^{\mathrm{e}}$ L.C. International conference, Kent, U.S.A. (1976).

[2a] Proust, J. E., Perez, E., Ter Minassian Saraga, L., Colloid Polymer Sci. 254 (1976) 672 et sous presse.

[2b] Proust, J. E., Perez, E., J. Physique Lett. 38 (1977) L-91.
[3] Langevin, D., J. Physique 37 (1976) 755.

[4] Perez, E., Proust, J. E., Ter Minassian Saraga, L., 6e L.C. International conference, Kent, U.S.A. (1976) et en cours.

[5] Williams, C., Thèse, Paris (1976).

[6] Kleman, M., Williams, C., Phil. Mag. 28 (1973) 725.

[7] Pfrf7. E.. Thèse 3e cycle Paris VII (1977). 